\title{
Secondary School Students' English Writing Aided by Spelling and Grammar Checkers
}

\author{
Odette Radi \\ La Trobe University, Australia
}

\begin{abstract}
This paper presents phase one of a study aimed at investigating how and why secondary school students use computer tools, such as spelling and grammar checkers, to aid them in their English writing and how their patterns of use relate to their English literacy. The study was prompted through close observations over many years, on how students use computers to support their writing. The observation indicated that while some students make a lot of use of computers, they still struggle to read and write in English. The research involved testing and surveying sixty-five Year 8 students. The test results obtained are compared with students' responses to the survey. The survey questionnaire contained open ended questions for the students to respond reflectively and to evaluate their uses of computer tools.
\end{abstract}

Keywords: Students, computer use, spelling and grammar checkers, literacy, secondary education, cognition, curriculum, social demands and practices.

\section{Introduction}

The study consisted of two phases: Phase one was screening procedures and Phase two involved participation of six case studies. This paper presents phase one of the study which began with a sample of sixty-five Year 8 students, aged 13 to 14 years old in a co-educational, intermediate metropolitan school in Victoria, Australia. The study began by measuring the students' use of computers at home and at school as well as gathering data on their performances on selected standardised English literacy tests. In analysing these results, no other factors have been compared with or looked at such as cultural background, family life, screen time, sports activities, background history, nor were any comparisons made to other studies in the same community.

The paper compares the Australian Council for Educational Research (ACER) [1] tests results to the students' survey responses and their use of computer tools in their domestic and school environments. The findings will examine whether the increased use of computer tools has any influence/impact on the students' development in language literacy skills.

\section{Purpose of the Study}

The focus of the study has stemmed from personal observation over the years as a classroom teacher in the areas of Computer Studies and Humanities. With the 
increased use of computers in both domestic and school environments, students have displayed more interest in the use of computers. There has been a shift in emphasis from reading the traditional printed text to include the use of the microelectronic medium. This shift had a bearing on the development of literacy (vocabulary, comprehension and writing) skills that for some students now reflect a decrease in the influence of the printed texts and conventions.

The transformation of educational activities into the electronic medium has sometimes been overwhelming to the young, their families and policy makers. "What has stimulated policy change has been the beliefs about average education levels in the labour force and the claimed effect of these on economic performance" [2, p. 87]. Computers in the education and domestic environments have become a common place for both teaching and learning. What is needed is detailed attention to the ways in which computers are used by individuals and their acquisition of computer and language literacy skills.

\section{Acquiring Computer and Language Literacy Skills}

Acquiring computer and language literacy skills differ in their concepts, cognition, attainment and learning development. For example, language literacy acquisition is a life long learning skills which begins from birth and requires proficiency and input from the individual's surroundings. While, the computer literacy skills are acquired in a much less time than its counterpart. The same skills repetitively applied when the users operate the computer and its components as well as the ability to manipulate data using the required software applications. However, both literacy skills are equally important in contemporary society.

\subsection{Acquiring Computer Literacy Skills}

Computer literacy skills are basically acquired, in a less specialised level, by learning how to turn on a computer, start and stop simple software applications, to save and print documents. As computers advanced over the years, the skills acquired are the use of the alphanumeric keyboard and mouse handling, in conjunction with other computer components such as the scanner, iPod, digital camera and projector, CD-ROM, DVD and the explosion in software available for use, including Internet [3].

\subsection{Acquiring Language Literacy Skills}

Language literacy acquisition determines the relationship between cognitive skills (perception and memory) and reading skills (decoding and comprehension) and writing skills. The Literacy Dictionary [4, p. 282-283] defines both word recognition and word identification as "the process of determining the pronunciation and some degree of meaning of an unknown word". Thus, language literacy can range from 
"word identification, word recognition" and decoding to understanding the intent of the person who wrote the passage or influencing readers when the definition is applied to writing [5].

\title{
3.2.1 Computers as Cognitive Tools
}

In order to use computers as cognitive tools effectively in classrooms, it is necessary to change the curriculum, especially at the secondary level. It is readily apparent that there is considerable disagreement concerning the scale and pace of change that is liable to be associated with computers and language literacy acquisitions [6]. Computers have their benefits in areas like the workplace and the industry for mass production, fast communication and profits, but the employees still require the ability to read and write in order to function effectively and proficiently in their job. This proficiency needs to develop in school age students if they are to eventually attain the necessary skills in the language literacy.

\subsection{Literature Review - Similar Studies}

Studies were conducted and shown that the increased use of computers in both education system and domestic environment have had negative implications on language literacy development for school age children. Fuchs' and Wößmann's studies reported that "the computer availability and use at home ... [revealed] ... a negative relationship between home computers availability and student achievement". Their results suggest "having a computer at home and using it at school will almost certainly raise some computer skills at the expense of other skills" which negatively related to student performance in math and reading [7, p. 17-18]. Similar findings have been reported by Leino et al [8] whose study examined both traditional printed reading and Internet activities based on the data collected by the Programme for International Student Assessment (PISA) 2000 study. They reported that;

\begin{abstract}
Active traditional reading with a strong engagement in fiction is associated with a high level of reading literacy proficiency. ... In contrast, the lowest performance level was attained by the group of heavy digital readers, who typically almost never read traditional fiction or non-fiction. This suggests that if students tend to read electronic texts only, they will not reach a very high level of literacy - at least when assessed by using traditional texts and methods [8, p. 272].
\end{abstract}

By contrast to Leino el al's report, Ljungdahl and March stated that "[C]omputer tools [spell and grammar checkers] improve spelling and grammar accuracies. If the original work has been word-processed, errors picked up may be simple typographical (e.g. computers tend to correct hte to the automatically)," students should be encouraged "to take note of which spelling errors they make and why they might have made them" [9, p. 272]. 


\section{Research Methodology}

Quantitative method was used to correlate the results by testing and surveying Year 8 students and collecting data to eliminate some phenomena/ assumptions about students' computer use and English writing [10]. The number of students who consented to participate in the study was 65 out of 135 . There was a gender imbalance at Year 8 level in 2005; 80 boys and 55 girls. The number of boys at this level exceeded the number of girls. Consistent with this were 40 boys and 25 girls who returned the consent forms, sat the tests and answered the questionnaire.

The data for this paper was gathered by administering (1) ACER tests: Progressive Achievement Tests in Reading: Vocabulary and Comprehension (PAT - R) [1] and Developmental Assessment Resource for Teachers (DART - English) writing test [11]; and (2) a self-developed questionnaire that explored patterns of educational and domestic computer use, by Year 8 students. The research issue was whether there was a relationship between performance on standardized tests of English literacy and patterns of use of computer tools, spelling and grammar checkers.

\section{Results of the Study}

The ACER tests results consisted of word knowledge, literal and inferential comprehension of prose material and three pieces of writing. The graphs below display the results of the boys' and girls' performance on the three tests; reading vocabulary, reading comprehension and writing. The final analyses below are based on the performance of each gender.

\subsection{ACER Tests Results}

The overall average results are shown in Graph 1 below.

Graph 1. Average performance of boys and girls in Vocabulary, Comprehension and Writing tests

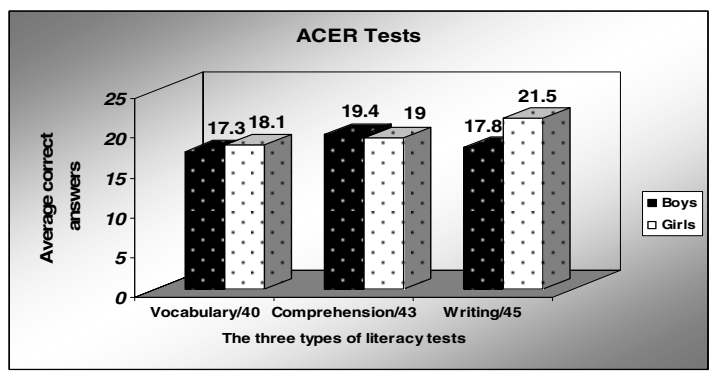

From examining Graph 1, the group performed differently on the vocabulary and writing tests. However, when independent group T-test was applied there was no statistical difference in the test results for vocabulary and comprehension between the boys and the girls. On the other hand, the writing test results showed the T-test for equality of means on the 2-tailed significant difference was .039 which was significant at $95 \%$ confidence 
interval that the girls did perform significantly better than the boys. In the next section, Graph 1's average tests results will be compared with the students' survey responses.

\subsection{Use of Spell Checker}

Data in Graphs 2 and 3 summarise the students' responses on how useful they find the spell and grammar checkers while they are composing their English writing pieces on the computer. Table 1 will also demonstrate later how the students apply their strategies and experiences with the use of computer tools.

Graph 2. The use of spell checker

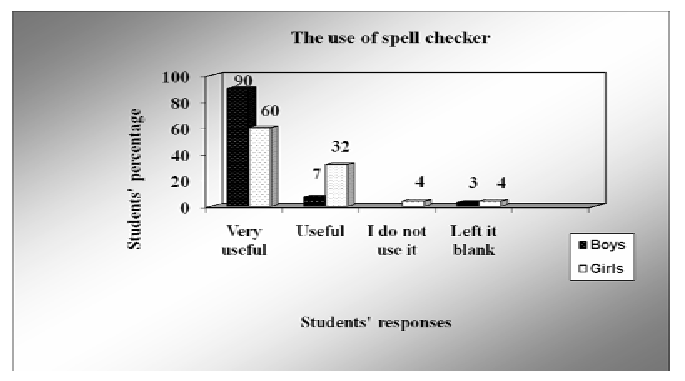

The majority of students indicated that they find the spell checker very useful. Graph 2 illustrates their responses of $97 \%$ of boys find the spell checker very useful to useful compared to $92 \%$ of the girls. Some of their general comments were: "When I am typing in Microsoft Word, the incorrect spellings are underlined with red squiggly lines which indicate that there are spelling errors". "The spell checker will help me correct them." "I do not have to worry about it". "I do not have to remember how to spell it correctly because next time it happened I will do the same thing by using the spell checker." Obviously, the pupils are aware of the computer prompts and its operational functions. They have no difficulty interacting with the tools in order to enhance the quality of their English writing.

\subsubsection{Computer-Pupil Interactions}

Computer-pupil interactions are often characterised as progressive. However, we do not have an understanding of how children use computer tools and how they apply their strategies and experiences to aid them in their language literacy skills. Consequently, the implications of using computers as cognitive tools will place the emphasis of learning on the mastery of certain thinking skills as mentioned by the participants.

\subsection{Spell Checker and Strategy for Word Replacement}

One of the open-ended questions in the survey asked students about how they go about choosing the right word from the spell checker's list to replace the underlined word(s) with red squiggly lines. Their responses are grouped in reflective and nonreflective [12] responses in order to understand their strategies. The students' responses are shown in Table 1. 
Table 1. Spell checker and strategy for word replacement

\begin{tabular}{|l|c|c|}
\hline Spell checker and strategy for word replacement & Boys \% & Girls\% \\
\hline Reflective response & & \\
\hline Look it up in the dictionary - look for the meaning & 10 & 32 \\
\hline Ask parents/someone & 11 & 8 \\
\hline By using the thesaurus as well & 3 & 12 \\
\hline From memory/general knowledge & 7 & \\
\hline The word that makes sense & 7 & \\
\hline You pronounce the word to see if it is right & 3 & 4 \\
\hline Recognise word & 3 & \\
\hline I read it & 3 & \\
\hline Subtotal & $\mathbf{4 7}$ & $\mathbf{5 6}$ \\
\hline Non-reflective response & & \\
\hline You click on the first one in the list & 18 & 16 \\
\hline Left it blank & 14 & 8 \\
\hline Take a guess & 12 & 12 \\
\hline I click ignore & 3 & 4 \\
\hline I do not know & 3 & 4 \\
\hline If it looks rite I will choose it & 3 & \\
\hline Subtotal & $\mathbf{5 3}$ & $\mathbf{4 4}$ \\
\hline
\end{tabular}

The purpose of Table 1 is to show that the students understand what the spell checker does. The majority of students find the spell checker very useful to useful (refer to Graph 2). The strategies they engage with in regards to the computer prompts differ in their reflective and non-reflective responses. Table 1 shows slightly more (girls) or less than half (boys) of the responses to the cue take on a reflective attempt to correct the underlined word with red squiggly lines.

\subsubsection{Reflective Group of Responses}

The way the reflective group responded was that $32 \%$ of girls and $10 \%$ of boys put into actions reflective and thoughtful strategies such as "look it up in the dictionary"; and $8 \%$ of girls and $11 \%$ of boys seek or "ask parents/someone" for help.

\subsubsection{Non-reflective Group of Responses}

The non-reflective group of students responded that they understand the computer prompts (e.g. the red and green squiggly lines when they appear on the screen), but they randomly choose different strategies to get rid of the lines. For example, $18 \%$ of boys and $16 \%$ of girls choose to "click on the first one in the list" without giving a thought about whether the word replacement is correct or not. Equal 12\% of boys and girls responded with "take a guess". This indicates that they are not quite sure of the correct word replacement/spelling. A small percentage responded "click ignore", "I do not know" and "if it looks rite I will choose it". This demonstrates that the students know how to use the computer tools but they do not know how to apply that experience into more general language literacy context. 


\subsection{Use of Grammar Checker}

Graph 3 illustrates that students are also relying on the use of the grammar checker. The data indicate that $85 \%$ of boys find it very useful to useful compared to $84 \%$ of girls. Graphs 2 and 3 indicate that the students are frequently using the computer tools when they are composing their English writing pieces. All students have the same access to computer equipment at school and in their domestic environment. They are comfortable in the use of the hardware, as well as the software applications. They find that the grammar checker suggests alternative sentence structures to enhance their writing. Students' reflection on the use of computers was that "we use the computer tools to correct the mistakes after we access the information that we need for our assignments, from the Internet and the compact disk". Obviously, the students understand how to use the microelectronic medium, how "to access the information, manipulate it, transform it and exchange it" [14, p. 34].

Graph 3. The use of grammar checker

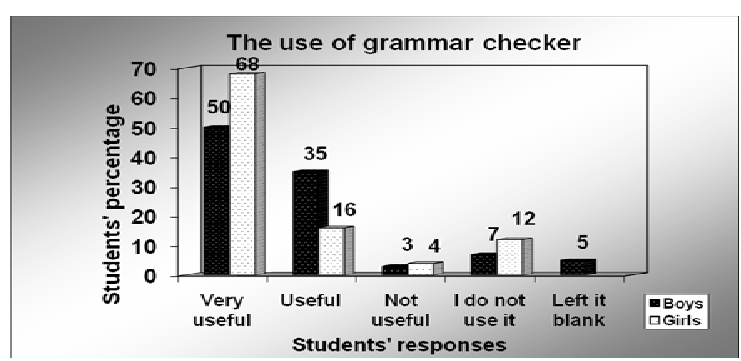

The ACER tests, the average results in Graph 1, revealed students performed below average in the three literacy tests. The average scores are 17 $18 / 40$ for vocabulary, equal $19 / 43$ for comprehension and $17-21 / 45$ for writing. The tests were based on traditional printed texts. Their responses to the survey on the usefulness of the spell (with autocorrect option) and grammar checkers (Graphs 2 and 3) may imply (including the estimated time spent using the computer) that the students are spending their time being as digital readers and composers [8, p. 262] and [9 p. 272]. The obvious question remains unanswered; if the students performed the literacy tests on the computer, would the results be different? The next section will look at the usefulness of the computer tools (spell and grammar checkers) as claimed by the participants, and its effect on the traditional printed literacy tests.

\subsection{ACER Test Results Mean and Minutes Per Week}

The results were compared to show whether the final results revealed any differences between the boys' and the girls' performance in the tests. Then the correlation coefficient distribution was used to measure the correlation outcomes between the students' responses of their estimated time (in minutes per week) spent using computers at home against the results of the ACER tests. The results of the correlation distribution are presented in Graph 4.

The results are not entirely consistent, but the dominant relationship is positive. For comprehension and writing, for both boys and girls and overall there is a positive relationship between minutes per week of computer use and scores on the tests. In other words, boys and girls who spend longer on the computer perform better on reading comprehension and writing. 
Graph 4. Boys and Girls overall test results mean and minutes per week

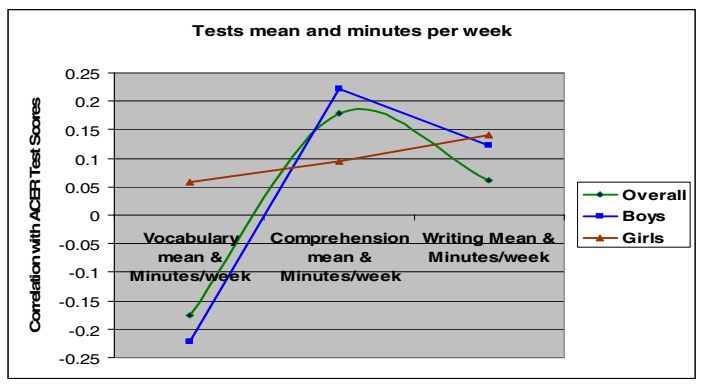

This pattern does not hold in its entirety for vocabulary. Here the results for the boys and girls are quite different. The results for the girls are the same as for comprehension and writing. Girls who spend longer on the computer performed better on the vocabulary test. However, there was a negative correlation for boys of -0.22 . More extensive use of computers by boys correlated negatively with vocabulary scores

leading to an overall correlation for the whole cohort of boys and girls to be negative $(-0.16)$. Since the overall performance of both boys and girls on the tests was at less than pass levels. Clearly, something was contributing to the overall low performance as shown in Graph 1 for both boys and girls, but these results suggest that for these particular students, with the exception of vocabulary for boys, greater use of computers correlated with better test scores.

\section{Conclusion}

The results of the study showed that the students are engaged with the use of computer tools (spell and grammar checkers) in both education and domestic environments. This emphasises that there has been a shift from traditional language literacy to computer-based literacy. This study has found that there is an indication of both positive and negative relationships between the two literacies.

The study showed that the students' responses indicated an extensive use of computers. Nevertheless, this is not fulfilling the functions of connecting the computer tools to the wider language literacy skills. The results in Graph 1 (literacy tests) compared to students' perception in Graphs 2 and 3 (spell and grammar checkers) indicate that the regular use of computer tools at Year 8 level has implications on the development of their language literacy skills. As demonstrated in Table $1,53 \%$ of boys and $44 \%$ of girls responded with non-reflective strategies when selecting the correct vocabulary word replacement from the spell checker's list while composing their English writing. Meanwhile, Graph 4 shows positive and negative outcomes in the correlation coefficient distribution between the results of the ACER tests results and the estimated time spent on computers per week at home. More extensive use of computers by boys correlated negatively in the vocabulary scores. The collected data from the survey also revealed that $50 \%$ of boys compared to $34 \%$ of girls use their personal computer for amusement purposes. The games played as indicated in the questionnaire include non-educational ones like racing cars, soccer, football and simulated arcade games. For them to become part of the same literate community of practice, they will need to be able to make use of computer tools to attain their spelling and grammar skills.

The primary and junior secondary students are still in the process of developing mentally and physically. If schools do not adapt to meet the needs in reading and 
writing, a growing number of parents could feel alienated by the inadequacies of schooling. Goss [15, p.15] states that "the issue is not computers versus literacy on the basis that the increased use of microelectronic tools still requires adequate literacy skills to enable students and others to interpret the messages, look for several possible meanings and also fully participate in reading and writing". My data is consistent with this. Some students reported strategies that showed them reflecting on the literacy choices that the computer prompted them. However, almost as many students reported a non-reflective response. The issue appears to be more than one of how reflection is brought into computer use rather than how computers damage literacy skills.

\section{References}

1. Australian Council for Educational Research: Progressive Achievement Tests in Reading: Comprehension and Vocabulary Tests Form 4, 3rd edn. ACER Press, Victoria (2001)

2. Lo Bianco, J.: Public Policy and Literacy Research Data: Will knowing led to Doing? In: Mclennan, W. (ed.) Aspects of Literacy: Assessed Skill Levels, Catalogue Number 4228.0. Australian Bureau of Statistics, Australia, pp. 80-88 (1997)

3. Winch, G., Holliday, M.: Reading. In: Winch, G., Johnston, R.R., March, P., Ljungdahl, L., Holliday, M. (eds.) Literacy: Reading, Writing and Children's Literature, 3rd edn., pp. 1-155. Oxford University Press, New York (2006)

4. Harris, T.L., Hodges, R.E.: The Literacy Dictionary: The Vocabulary of Reading and Writing. International Reading Association, U.S.A (1995)

5. Christensen, C.: Decoding: Reading Link is Transforming the Teaching of Literacy. The Enquiries into teaching of literacy report. Network Educational, Australia (December 2005)

6. Mandinach, E.B., Cline, H.F.: It Won't Happen Soon: Practical Curricular, and Methodological Problems in Implementing Technology-Based Constructivist Approaches in Classrooms. In: Lajoie, S.P. (ed.) Computers as Cognitive Tools: No More Walls, pp. 377-389. Lawrence Erlbaum Associates, New Jersey (2000)

7. Fuchs, T., Wößmann, L.: Computers and Student Learning: bivariate and Multivariate Evidence on the Availability and Use of Computers at Home and at School. CESIFO Working Paper No. 1321. Category 4: Labour Markets (November 2004), http: / /www.res.org.uk/econometrics/504.pdf

8. Leino, K., Linnaky, P., Malin, A.: Finnish Students' Multiliteracy Profiles. Scandinavian Journal of Educational Research 48(3), 251-270 (2004), http: / /www. informaworld.com/smpp_content=t713445993

9. Ljungdahl, L., March, P.: Spelling Skills in the Classroom. In: Winch, G., Johnston, R.R., March, P., Ljungdahl, L., Holliday, M. (eds.) Literacy: Reading, Writing and Children's Literature, 3rd edn., pp. 254-281. Oxford University Press, New York (2006)

10. Gall, M.D., Gall, J.P., Borg, W.R.: Educational Research: An Introduction, 7th edn. Allyn and Bacon, New York (2003)

11. Forster, M., Mendelovits, J., Masters, G.: Developmental Assessment Resource for Teachers: DART English. The Australian Council for Educational Research Ltd., Victoria (1994)

12. Martin, A.: In: Martin, A., Rader, H. (eds.): Information and IT Literacy: Enabling learning in the $21^{\text {st }}$ century, pp. 3-23. Facet Publishing, London (2003)

13. Harris, F.J.: I Found it on the Internet: Coming of Age Online. American Library Association, Chicago (2005)

14. Goss, S.: Learning More About - Literacy and Access: Much More than Reading and Writing. The Age Newspaper. Education section, 15 (November 25, 1997) 